(at 1-minute interval) during surgery, were collected. Using the preoperative noninvasive and intraoperative invasive systolic blood pressure values, we calculated the average real variability (ARV), known as a reliable representation of time series blood pressure variability. Associations between the ARV index and survival outcomes were investigated.

Results: In total, 441 patients were included. Using the integer close to the median as a cut-off value for the ARV, we found that the high-ARV group $(\geq 8 ; n=220)$ showed worse progression-free survival (PFS) than the low-ARV group $(<8$; $\mathrm{n}=221$ ) (median $=82.8 \%$ vs. $89.6 \%$; $=0.020$ ). In multivariate analysis adjusting for confounders, ARV $\geq 8$ was identified as an independent poor prognostic factor for PFS (adjusted hazard ratio $[\mathrm{HR}]=1.887$; 95\% confidence interval $[\mathrm{CI}]=1.158-3.076$; $\mathrm{p}=0.011)$. In the subgroup of open RH $(\mathrm{n}=238), A R V \geq 8$ was associated with significantly worse PFS (adjusted HR=2.402; 95\% CI=1.119-5.155; $\mathrm{p}=0.024)$. In contrast, in the subgroup of MIS RH ( $n=238)$, PFS did not differ by the ARV index.

Conclusion: The ARV index, indicating intraoperative hemodynamic instability, might be a novel prognostic biomarker for disease recurrence in early cervical cancer patients who receive primary open $\mathrm{RH}$, not MIS RH.

Oral (OC3)

Cervical Cancer

https://doi.org/10.3802/jgo.2021.32.S1.0C3

\section{Comparison of locally advanced cervical cancer treatment guidelines in Asia}

\author{
David Tan, ${ }^{1, *}$ Bradley J. Monk, ${ }^{2}$ Jitender Takyar, ${ }^{3}$ \\ José David Hernández Chagüi, ${ }^{4}$ Takayuki Enomoto, ${ }^{5}$ Eric \\ Pujade-Lauraine $^{6}$ \\ 'National University Cancer Institute, Singapore \\ (david_sp_tan@nuhs.edu.sg) \\ ${ }^{2}$ University of Arizona College of Medicine, Tucson, AZ, USA \\ ${ }^{3}$ Parexel International, Mohali, India \\ ${ }^{4}$ AstraZeneca, Gaithersburg, MD, USA \\ ${ }^{5}$ Niigata University Graduate School of Medical and Dental Sciences, Niigata,
} Japan 6ARCAGY-GINECO, Paris, France

Objective: A previous global systematic literature review (SLR) assessing recommended treatment for locally advanced cervical cancer (LACC) revealed international consensus on the use of concurrent chemoradiotherapy (CCRT) as standard of care (SoC) for stage IIB-IVA LACC. However, recommendations for stage IB2-IIA LACC varied. We present a subanalysis of Asian guidelines to determine if recommended LACC treatment is consistent across Asia.

Methods: The most recent English-language cervical cancer treatment guidelines and consensus statements were identified from literature databases (1999-2020), national authority websites, and bibliographies. For comparison, additional, nonEnglish, Asian country guidelines were translated. Results: Eleven guidelines from 5 Asian countries were reviewed; 10 were last updated between 2013-2021, 1 was undated. Seven guidelines provided treatment recommendations by disease stage using International Federation of Gynecology and Obstetrics (FIGO) 2009 staging criteria. For stage IB2-IIA2 disease, surgery, cCRT, or RT alone was recommended. Cisplatin-based cCRT followed by brachytherapy was SoC for suitable patients with stage IIBIVA LACC among all Asian guidelines, except Japan, where radical hysterectomy or cCRT was recommended for stage IIB. For stage IVA disease, 2 Indian guidelines (ICMR and NCGI) discussed the use of palliative treatments versus curative intent therapy. Non-SLR guidelines from Vietnam and Malaysia were consistent with this consensus.

Conclusion: In line with global SLR findings, consensus on cCRT as primary treatment for stage IIB-IVA LACC was recommended by Asian guidelines. For stage IB-IIA LACC, recommendations varied between radical hysterectomy and cCRT, or cCRT/RT alone. Alignment of guidelines with FIGO 2018 staging criteria may reduce variation in recommended treatment for early-stage LACC.

Funding: AstraZeneca.

Oral (OC4)

Cervical Cancer

https://doi.org/10.3802/jgo.2021.32.S1.0C4

\section{Adjuvant pelvic radiation versus observation in intermediate-risk early-stage cervical cancer patients following radical surgery: a propensity score analysis}

Thunwipa Tuscharoenporn, Tanarat Muangmool, Kittipat Charoenkwan*

Chiang Mai University, Chiang Mai, Thailand (kittipat.c@cmu.ac.th)

Objective: To compare survival outcomes, posttreatment complications, and quality of life of early-stage cervical cancer patients classified as intermediate-risk group following primary radical surgery between the patients who received adjuvant pelvic radiation and those without adjuvant treatment. Methods: Two hundred and thirty stage IB-IIA cervical cancer patients who had radical hysterectomy and pelvic lymphadenectomy from January 2003 to December 2018 and were classified as having intermediate-risk according to the Sedlis's criteria were included. The participants were divided 
into two groups-adjuvant pelvic radiation (116 patients) and no adjuvant treatment (114 patients). The propensity scores were employed to balance the 2 study groups with respect to distribution of confounding factors. The primary outcomes were disease-free survival (DFS) and overall survival (OS). The secondary outcomes included treatment complications and quality of life.

Results: The DFS were comparable between the groups with the adjusted hazard ratio (aHR) 0.46 (95\% confidence interval $[\mathrm{CI}]=0.18-1.16 ; \mathrm{p}=0.1)$ for adjuvant radiation. Similarly, the OS were not significantly different with the aHR 1.75 (95\% $\mathrm{CI}=0.63-4.90 ; \mathrm{p}=0.3$ ) for adjuvant radiation. Serious side effects were reported in 4 patients, 3 in the adjuvant radiation group ( 2 lymphedema and 1 enterovesical fistula) and 1 in the no adjuvant treatment group (lymphedema). Quality of life scores were not significantly different.

Conclusion: The survival benefit of adjuvant pelvic radiation for intermediate-risk early-stage cervical cancer could not be demonstrated in this study.

Oral (OC5)

Cervical Cancer

https://doi.org/10.3802/jgo.2021.32.S1.0C5

\section{Therapeutic role of conization before radical hysterectomy in patients with early cervical cancer}

\section{Ji Song Min, ${ }^{1}$ Ki Hyeon Song, ${ }^{2}$ Chel Hun Choi,, Tae-Joong Kim, ${ }^{1}$ Jeong-Won Lee, ${ }^{1}$ Yoo-Young Lee ${ }^{1, *}$ \\ 'Samsung Medical Center, Seoul, Korea (yooyoung.lee@samsung.com) ${ }^{2}$ Sungkyunkwan University School of Medicine, Seoul, Korea}

Objective: The aim of this study is to investigate the role of conization prior to radical hysterectomy in patients with early cervical cancer.

Methods: Patients who had radical hysterectomy for early cervical cancer at Samsung medical center from 2001 to 2020 were eligible. Patients were divided into 2 groups; patients with pre-operative conization or patients who had biopsy.

Results: A total of 1,826 patients were included; 294 patients with pre-operative conization (16.1\%, group 1$)$, and 1,532 patients without conization (83.9\%, group 2). 255 patients in group 1 showed resection margin positives after conizations. Patients in group 1 were younger ( 46 years vs. 48 years, $\mathrm{p}<0.001)$. Non-squamous histology $(29.9 \%$ vs. $22.8 \%, \mathrm{p}=0.014)$ and bulky cervical mass (>4 cm, $16.9 \%$ vs. $4.1 \%$, p<0.001) were more frequent in group 2. Laparotomy was more popular in group 2 (70.5\% vs. $51.4 \%, \mathrm{p}<0.001)$, the proportion of type of radical hysterectomy was similar (type $3,98.2 \%$ vs. $97.6 \%$, $\mathrm{p}=0.472)$. On final pathology after radical hysterectomy, larger tumor size, depth of invasion more than $1 / 2$, lymphovascular space invasion positive, pelvis lymph node (LN) positive, para-aortic LN positive, microscopic parametrial invasion were more frequently observed in group 2 . When adjusting these factors in Cox model, patients with pre-operative conization showed survival benefit in progression free survival (hazard ratio $[\mathrm{HR}]=0.709 ; 95 \%$ confidence interval $[\mathrm{CI}]=0.480-1.045$; $\mathrm{p}=0.082$ ) and in overall survival (OS; HR $=0.449 ; 95 \% \mathrm{CI}=0.276-$ $0.278 ; \mathrm{p}<0.001)$.

Conclusion: Our data suggest the therapeutic role of conization before radical hysterectomy in early cervical cancer which showed better OS.

Oral (OC6)

Cervical Cancer

https://doi.org/10.3802/jgo.2021.32.S1.0c6

\section{Definitive radiotherapy for cervical adenocarcinoma: outcomes and immune- related factors}

\author{
Yuhei Miyasaka,' Yuya Yoshimoto, ${ }^{2, *}$ Kazutoshi Murata, ${ }^{3}$ \\ Ken Ando, ${ }^{4}$ Takeshi Ebara, ${ }^{5}$ Noriyuki Okonogi, ${ }^{3}$ Hayato Ikota, ${ }^{6}$ \\ Hideaki Yokoo, ${ }^{7}$ Tatsuya Ohno, ${ }^{4}$ Takashi Nakano ${ }^{8}$ \\ 'Gunma University Heavy Ion Medical Center, Maebashi, Japan \\ ${ }^{2}$ Department of Radiation Oncology, Fukushima Medical University, \\ Fukushima, Japan (yyoshimo@fmu.ac.jp) \\ ${ }^{3}$ QST Hospital, National Institutes for Quantum and Radiological Science \\ and Technology, Chiba, Japan \\ ${ }^{4}$ Department of Radiation Oncology, Gunma University Graduate School \\ of Medicine, Maebashi, Japan \\ ${ }^{5}$ Department of Radiation Oncology, Kyorin University, Tokyo, Japan \\ ${ }^{6}$ Department of Diagnostic Pathology, Gunma University Hospital, \\ Maebashi, Japan \\ ${ }^{7}$ Department of Human Pathology, Gunma University Graduate School of \\ Medicine, Maebashi, Japan \\ ${ }^{8}$ National Institute for Quantum and Radiological Science and Technology, \\ Chiba, Japan
}

Objective: Data on prognosis and immune-related factors specific for cervical adenocarcinoma (CA) treated with definitive radiotherapy (RT) were insufficient. To evaluate these, we conducted a multi-institutional retrospective analysis.

Methods: Patients with CA treated with definitive RT comprising external beam RT and intracavitary brachytherapy were included. Pretreatment biopsy samples were stained with programmed death-ligand 1 (PD-L1) and CD8 antibodies. Associations of these molecules with clinical outcomes were evaluated.

Results: A total of 71 patients were included in this study. Median follow-up period was 37 months (range, 5-194). 